\title{
Editorial
}

\section{¿Qué casos clínicos se publican en la revista Odontología Sanmarquina?}

\author{
What clinical cases are \\ published in Odontología \\ Sanmarquina journal?
}

Manuel Antonio Mattos-Vela 1,a,c, Yuri Castro-Rodríguez 1,b,d

${ }^{1}$ Universidad Nacional Mayor de San Marcos, Facultad de Odontología. Lima, Perú.

a Doctor en Estomatología.

${ }^{b}$ Maestro en Educación.

c Editor general de la revista Odontología Sanmarquina.

${ }^{d}$ Editor adjunto de la revista Odontología Sanmarquina.

\section{Correspondencia:}

Manuel Antonio Mattos-Vela: mmattosv@unmsm.edu.pe Universidad Nacional Mayor de San Marcos, Facultad de Odontología. Calle Germán Amézaga 375. Lima 1, Perú.

ORCID: 0000-0002-5701-1961

Coautor:

Yuri Castro-Rodríguez: yuricastro_16@hotmail.com ORCID: 0000-0002-9587-520X

Recibido: 04/10/20

Aceptado: 05/10/20

Publicado: $16 / 11 / 20$
Nuestra revista ha experimentado en los últimos años un incremento en los manuscritos que llegan para revisión, en especial la sección de casos clínicos. El 2018 se recibió 25, el 2019, 29 y el 2020 fueron 44. Agradecemos mucho a los autores por su interés en publicar en nuestra revista. Considerando solo el 2020, los manuscritos del tipo "reporte de caso" procedieron de 10 países de Iberoamérica, siendo los más frecuentes México, Perú y Ecuador; ocho fueron aprobados para publicación, 29 rechazados $(66 \%)$ y siete se encuentran en proceso de revisión.

Frente al aumento de casos clínicos recibidos por la revista, queremos comunicar que el Comité editor ha tomado la decisión de designar a uno de sus editores como encargado de la primera revisión de estos manuscritos para evaluar el cumplimiento estricto de las directrices de la revista y la originalidad del mismo, cumplido estos requisitos recién el documento ingresará al proceso de revisión por pares.
Las directrices para autores de Odontología Sanmarquina establecen que los casos clínicos "consisten en la descripción de uno o más casos clínicos de especial interés, así como de nuevas técnicas quirúrgicas o variantes de aquella, análisis de resultados obtenidos con determinada técnica o en el tratamiento de una patología infrecuente o compleja. El número máximo de autores firmantes será de cinco... Los gráficos deben facilitar la comprensión del caso a reportar. El texto principal de los reportes clínicos debe ser organizado en: Introducción, Reporte del caso (debe incluir el diagnóstico, tratamiento, seguimiento y resultados), Discusión y Referencias bibliográficas" ${ }^{1}$. Además, se deben considerar las directrices $\mathrm{CARE}^{2}$ al momento de redactar este tipo de artículos.

No todos los casos clínicos que se atienden en la consulta particular, hospitales o facultades de Odontología (Estomatología) merecen ser publicados como un "reporte de caso clínico". Para que pueda ser aceptado en una revista 
se requiere que el informe refleje un aspecto inédito, inusual o raro de la práctica clínica o la práctica forense. Requiere que el "evento anecdótico" del caso amerite un nuevo diagnóstico, nuevo tratamiento, introducción de un biomaterial, solución de complicaciones, complicaciones no esperadas en la evolución del paciente, signos y síntomas poco usuales o no descritos en la literatura así como procedimientos novedosos para la identificación de un hallazgo (propio de las ciencias forenses). En este sentido, no ameritan ser publicados como casos clínicos lesiones tumorales que son frecuentes en la práctica hospitalaria, diagnósticos usuales como periodontitis o caries dental, procedimientos rutinarios como la colocación de implantes dentales o el tratamiento ortodóntico. Sí ameritarían ser valorados para su publicación: modificaciones a la terapia convencional, signos y síntomas inusuales de un diagnóstico conocido, complicaciones y solución de un tratamiento que se esperaría no tenga tales complicaciones, etc.

Múltiples manuscritos del tipo "reporte de caso" que han sido remitidos en el último ańo a la revista Odontología Sanmarquina lamentablemente han sido rechazados por no carecer de esa "inusualidad" al momento de reportar el evento anecdótico. Algunos de ellos han sido rechazados por adolecer de un seguimiento clínico apropiado cuando se realizan tratamientos odontológicos. Por lo general los reportes clínicos que se hayan basado en tratamientos requerirán de un control o seguimiento clínico puesto que se tendrá que evidenciar resultados luego de la intervención. Los tiempos de estos controles variarán según el caso; así por ejemplo para valorar la cicatrización de los tejidos blandos periodontales se requerirá un seguimiento mínimo de dos meses mientras que para valorar las modificaciones de casos relacionados a la terapia de periodontitis requerirán evaluaciones en un tiempo mínimo de seis meses. Los casos clínicos que presentan seguimientos de semanas, un mes, o solo un control posoperatorio no ameritan su publicación pues carecen del seguimiento necesario para valorar los resultados de tratamiento.

Antes de redactar el manuscrito recuerde revisar si su caso clínico no ha sido ya publicado, puesto que le restará lo novedoso. Para esto es recomendable consultar con especialistas en el tema y buscar en bases de datos. Por ejemplo: supongamos que en nuestra consulta privada utilizamos el propóleo peruano como irrigante para la terapia de la periodontitis, nuestro caso luego de seis meses de seguimiento reportó resultados favorables en la reducción de las bolsas periodontales y deseamos preparar el manuscrito para que pueda ser publicado; al realizar las consultas con especialistas valore las críticas de expertos reconocidos y que tengan experiencia publicando reportes de caso. También considere analizar bases de datos (por ejemplo: SciELO) e insertar los términos "propóleo" y "periodontitis", se encuentran 125 artículos por lo que nos percatamos que el tema no es tan novedoso como nosotros creíamos.

Frente a estos casos es mejor no continuar con el proceso de redacción del manuscrito puesto que no será un tema novedoso. Sin embargo, puede que los estudios o casos coincidentes no hayan sido publicados en el contexto nacional, en esos casos sí es posible redactarlo y presentarlo a la comunidad científica. Considere valorar qué casos clínicos ya ha publicado la revista que ha escogido puesto que si la revista ya ha publicado casos similares, las probabilidades de que su reporte de caso sea publicado serán mínimas.

La revista Odontología Sanmarquina valora los reportes de caso con los mismos criterios de calidad que los demás tipos de manuscritos. La evaluación de originalidad se realiza a través del software Ithenticate, considerándose original los documentos con un máximo de $10 \%$ de similitud, aplicando tres filtros al análisis (se excluyen: textos entrecomillados, bibliografía y frases menores de 40 palabras reportadas como similitud).

Hacemos un llamado a todos los autores que piensan enviar un caso clínico para ser evaluado por nuestra revista que tengan en cuenta estas consideraciones para evitar rechazos anticipados, es decir, en la primera revisión. A nuestros lectores queremos decirles que estamos comprometidos en mantener altos estándares en el proceso de revisión editorial ${ }^{3,4}$, de tal manera que seleccionaremos y publicaremos los mejores reportes de caso, que sean relevantes y ofrezcan un aporte y guía a la práctica clínica.

\section{Referencias bibliográficas}

1. Universidad Nacional Mayor de San Marcos [Internet]. Lima: UNMSM; 2020 [Consultado el 04 de octubre del 2020]. Odontología Sanmarquina. Disponible en: https://revistasinvestigacion.unmsm.edu.pe/index.php/ odont

2. CARE. Case report guidelines. [Internet]. [Consultado el 04 de octubre del 2020]. Disponible en: http://www. care-statement.org

3. Mattos-Vela MA, Cuadrao Zavaleta L. Revista al servicio de la comunidad científica [editorial]. Odontol Sanmarquina. 2017;20(2):37-38. DOI: 10.15381/os. v20i2.13976.

4. Mattos-Vela MA, Moromi Nakata H. El editor en Odontología Sanmarquina [editorial]. Odontol Sanmarquina. 2019;22(2):78-79. DOI: 10.15381/os.v22i2.16216. 